\title{
Comparison of Various Phenotypic Methods in Detection of Carbapenemases and Metallo-Beta-Lactamases in Carbapenem Resistant Clinical Isolates of Acinetobacter Species at a Tertiary Care Centre in North India
}

\author{
Ankur Goyal ${ }^{1 *}$, Neha K Mani ${ }^{1}$, Renu Chahar ${ }^{1}$, Ankita Soni ${ }^{1}$ and Sapna Goyal ${ }^{1}$
}

Department of Microbiology, Sarojini Naidu Medical College, Agra, Uttar Pradesh, India

*Corresponding author

\section{Keywords \\ Carbapenem resistance, Metallo- beta-lactamases (MBLs), Enzyme carbapenemases, Modified Hodge Test (MHT), Disc Potentiation Test (DPT), EDTA Disc Synergy (EDS) \\ Article Info \\ Accepted: 26 December 2017 Available Online: 10 January 2018}

\section{A B S T R A C T}

Carbapenem resistance has become a worldwide issue and a major reason of concern for the treating physicians. Plasmid mediated metallo-beta-lactamases (MBLs) as well as oxacillinases (OXA) are responsible for carbapenem resistance. Various phenotypic as well as genotypic screening methods are used for detecting carbapenemases and MBLs. The strains were first and foremost screened for carbapenem resistance by Kirby-Bauer Disc Diffusion method. The resistant strains were detected for the production of carbapenemases by Modified Hodge Test (MHT) followed by MBL detection using Disc Potentiation Test (DPT) and EDTA Disc Synergy (EDS) test simultaneously. Our study emphasizes on the detection of carbapenem resistant Acinetobacter sps. emerging due to MBL production, and also for understanding the impact of MBL production in carbapenem resistant strains. Among the 100 clinical isolates of carbapenem resistant Acinetobacter sps. subjected to the phenotypic detection of carbapenemases and metallo-beta-lactamases, carbapenemases production was found to be 86 per cent by MHT, MBL production as 43 per cent and 86 per cent by DPT and EDS respectively. Thus, according to our study, EDS was considered to be more sensitive and reliable phenotypic test for MBL detection in our isolates.

\section{Introduction}

Acinetobacter sps. are Gram negative, aerobic, non-fermenting bacteria playing a vital role in hospital acquired infections. Due to various obstacles in the treatment and control of the pathogen, it continues to be a major threat (Giamarellou $\mathrm{H}$ et al., 2008). Resistance for the $\beta$-lactam antibiotics has become widespread among Acinetobacter strains. There are several factors contributing for the carbapenem resistance such as hydrolysis by $\beta$-lactams (MBLs), lack of drug penetration due to porin mutation, loss of several outer membrane proteins and efflux mechanisms (Walsh TR et al., 2002).

Resistance due to hydrolysis by $\beta$-lactamase enzymes is a major cause of carbapenem resistance worldwide. Many times, multiresistant and PAN resistant Acinetobacter sps. have become a threat to any hospital in current 
antibiotic era. Therefore, rapid, simple and cost effective screening tests are adopted for identification of MBL and carbapenemases. Various phenotypic tests like EDTA (EDS) test (Lee et al., 2001), MBL E-test (Walsh TR et al., 2002), EDTA based microbiological assay (Marchiaro et al., 2005), DPT (Collee et $a l ., 2003$ ) are used for identification of MBLs and Modified Hodge Test (MHT) for carbapenemases.

Hence, the present study was undertaken to isolate Acinetobacter sps. resistant to carbapenems and study the production of MBLs and carbapenemases by various phenotypic methods. We have also compared various methods in the present study.

\section{Materials and Methods}

The study was conducted in department of microbiology of a tertiary care centre of North India. A total of 100 consecutive, nonduplicate, clinical strains of Acinetobacter sps. were isolated from various samples such as endotracheal aspirate, blood and pus respectively. These isolates were subjected to various phenotypic methods for detection of MBL and carbapenemases.

\section{MHT}

The carbapenem resistant strains were at first subjected to MHT for detecting carbapenemases production. The quality strain used is Escherichia coli_ATCC (American Type Culture Collection) 25922. This E.coli ATCC 25922 strain is adjusted to 0.5 McFarland's standard and inoculated on the surface of Muller-Hinton Agar (MHA) using a sterile cotton swab. After drying, a $10 \mu \mathrm{g}$ meropenem/imipenem disc was placed at the centre of the plate and the strains to be analyzed were streaked from the edge of the disc to the edge of the plate in four different directions. The plate was then incubated overnight at $37^{\circ} \mathrm{C}$. Presence of a clover leaf shaped zone of inhibition along the growth of the test strain was considered as positive for carbapenemases production (Noyal MJC et al., 2009) (Fig. 1).

All strains were further subjected to Disc Potentiation Test (DPT) and EDTA Disc Synergy (EDS) test for the detection of MBLs.

\section{DPT}

For detecting MBLs in carbapenem resistant isolates using DPT, the test organism was first adjusted to $0.5 \mathrm{McF}$ arland's opacity standards and inoculated on MHA plate. Two $10 \mu \mathrm{g}$ imipenem discs, one containing $750 \mu \mathrm{g}$ EDTA, obtained from Hi-Media Mumbai, were placed on the inoculated plate and incubated for 24 hours at $37^{\circ} \mathrm{C}$. The zones of inhibition around imipenem disc alone and imipenem-EDTA disc were recorded. An increase in the zone of inhibition of at least $7 \mathrm{~mm}$ around the imipenem-EDTA disc as compared to imipenem alone was considered as positive for MBL production (Krista $\mathrm{L}$ et al., 2006) (Fig. 2).

\section{EDS}

MBL detection by EDS is done by simultaneous testing of two different $\beta$ lactams imipenem and ceftazidime in carbapenem resistant strains (Lee et al., 2001). A $0.5 \mathrm{M}$ EDTA solution was prepared by dissolving 186.1 gms of Disodium EDTA. $2 \mathrm{H}_{2} \mathrm{O}$ (obtained from SRL) in $1000 \mathrm{ml}$ of distilled water. The $\mathrm{pH}$ is adjusted to 8.0 using $\mathrm{NaOH}$ (obtained from MERK) and was sterilized by autoclaving (Yong $\mathrm{D}$ et al., 2002). An overnight culture of the test isolate is adjusted to a turbidity of 0.5 McFarland standard (Krista et al., 2006) and spread on the surface of the MHA plate. A $10 \mu \mathrm{g}$ imipenem disc and $30 \mu \mathrm{g}$ ceftazidime disc is placed on the agar. A blank disc $(6 \mathrm{~mm}$ in diameter, 
Whatmann filter paper no. 1) was kept on the inner surface of the lid of the MHA plate and $10 \mu \mathrm{l}$ of $0.5 \mathrm{M}$ EDTA was added to it. This EDTA disc was then transferred to the surface of the agar and kept $10 \mathrm{~mm}$ edge to edge apart from the imipenem or ceftazidime disc. After incubating overnight at $37^{\circ} \mathrm{C}$, the presence of an expanded growth inhibition zone between the two discs was interpreted as positive for MBL production (Fig. 3).

\section{Results and Discussion}

Among the total 100 carbapenem resistant clinical isolates of Acinetobacter sps., 96 per cent were isolated from the endotracheal aspirate, 2 per cent from pus and 2 per cent from blood respectively.

In our study, 100 per cent strains were identified as enzyme producers, either as carbapenemases or as metallo-betalactamases.

Out of the total carbapenem resistant clinical isolates, 86 per cent $(86 / 100)$ of the strains were found to be positive for carbapenemases production by MHT. Whereas, 14 per cent of the strains not detected by MHT for carbapenemases production, were all detected as MBL producers by EDS and 29 per cent (4/14) of these MHT negative strains were reported as MBL producers by DPT as well.

In the present study, EDS reported 86 per cent $(86 / 100)$ of the strains positive for MBL production. However, total $14 \mathrm{MBL}$ negative strains were all positive for carbapenemase production by MHT. We used two drugs ceftazidime and imipenem to see MBL production by EDS method. On comparing individual drugs, 9 per cent of the strains showed synergetic effect only by ceftazidme and in 67 per cent of the strains, synergetic effect is shown by only imipenem. However, 24 per cent of the isolates showed synergetic effect by both ceftazidme and imipenem. Intrestingly, 85 per cent (12/14) carbapenemase negative strains by MHT were found as MBL producers by imipenem in EDS.

DPT using imipenem detected only 48 per cent (48/100) of the isolates as MBL producers. Out of 52 per cent undetected by DPT, $76.9 \%$ were detected as MBL producers by EDS and $80 \%$ as carbapenemases producers by MHT. An important observation that authors feel, is that despite using imipenem and imipenem EDTA in DPT, those strains which were negative by DPT, most of these strains $73 \%$ (38/52 strains) showed MBL production by EDS method using imipenem and freshly prepared EDTA discs. Therefore, we can assume that commercially available imipenem-EDTA disc may not contain right amount or right potency of EDTA solution in the disc. Although the sensitivity of DPT was the least, but interestingly, DPT was able to detect $14 \%$ (2/14) isolates as MBL producers that were reported negative by EDS.

Carbapenems have a wide spectrum of antibacterial activity, and are resistant to hydrolysis by most of the $\beta$-lactams including ESBL and Amp C $\beta$-lactamases (Payne DJ et al., 1997).

However, due to over and irrational use of carbapenems, an alarming increase in carbapenem resistance has been reported in many gram negative bacteria including Acinetobacter sps. (Walsh et al., 2005). Acinetobacter sps. has become a vital nosocomial pathogen in the last few years especially in ICU setting. 
Fig.1 Modified Hodge Test (MHT). Positive strain shows a 'cloverleaf shaped' zone of inhibition due to carbapenemase production

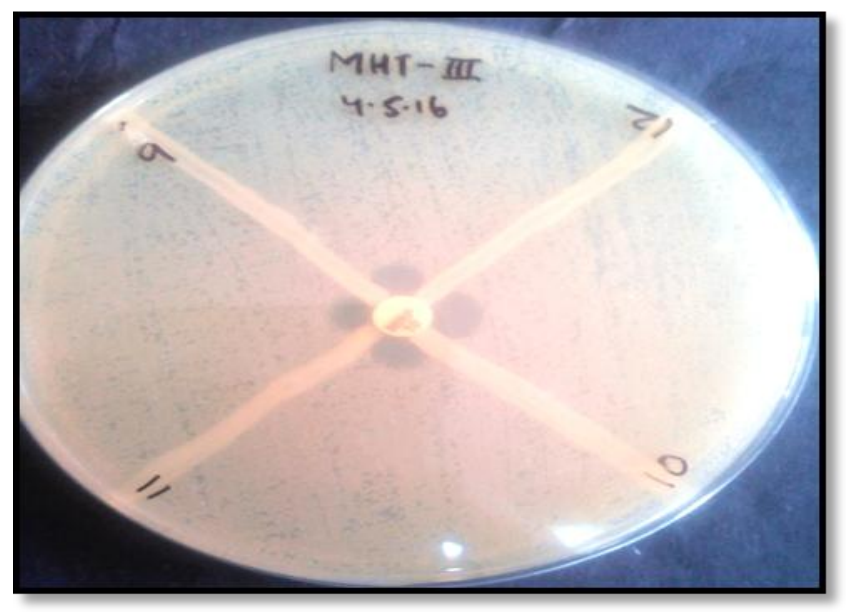

Fig.2 Disc Potentiation Test (DPT). An increase in the zone of inhibition of $>7 \mathrm{~mm}$ around the imipenem-EDTA disc as compared to imipenem alone is positive for MBL production

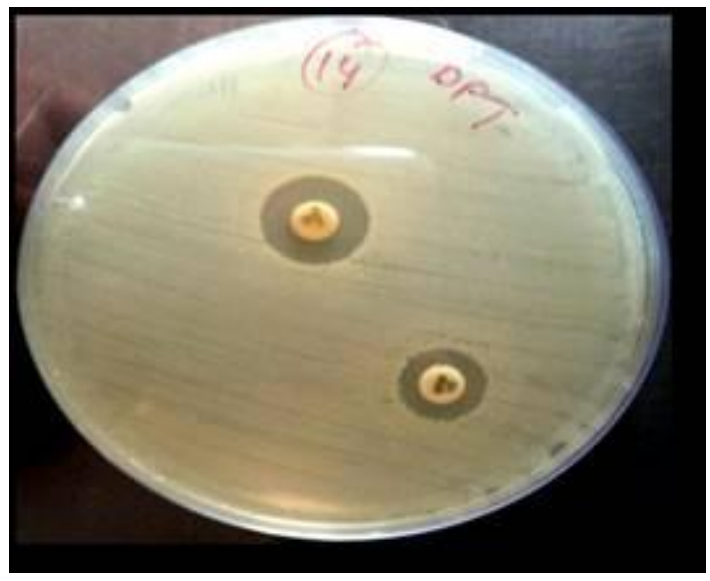

Fig.3 EDTA Disc Synergy Test (EDS). Positive strain shows a synergistic zone of inhibition between ceftazidime or imipenem disc and EDTA disc

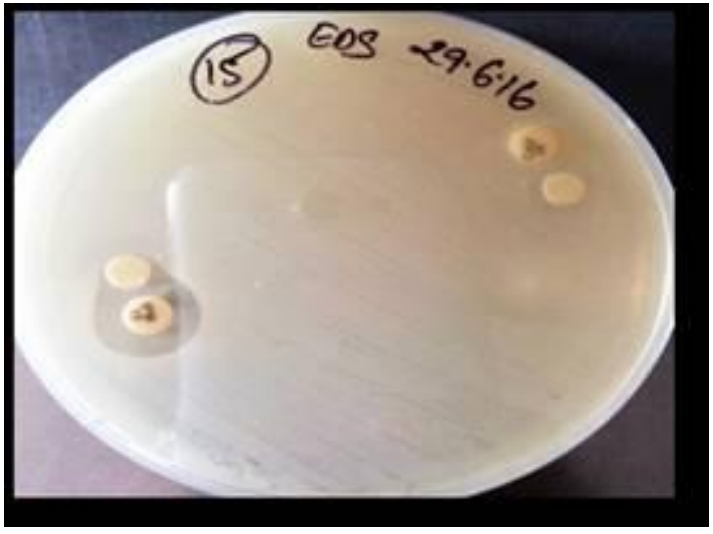


In the present study, we focused on carbapenem resistant Acinetobacter sps. for carbapenemases and MBL production in our set-up. Being a tertiary care centre, we also encountered high prevalence of carbapenem resistant bugs, especially Acinetobacter sps. We took 100 non- consecutive Acinetobacter $s p$. isolates in the present study. We found that $96 \%$ of our isolates were from the endotracheal secretions, which was in concordance to a study conducted by Muthuswamy et al., in Coimbatore (2012) where most of isolates were from respiratory secretions.

Many other studies (Duygu Aksoy et al., 2015, Aparna Shivaprasad et al., 2012, Jaggi et al., 2014) also reported high prevalence of Acinetobacter sp. in respiratory secretions. Therefore, we conclude that respiratory tract is a preferred site for Acinetobacter sp. infection. Most of our isolates were from ICU settings. A very similar observation was given by other authors like Sinha et al., (2007) and Richa Hans et al., (2015). They felt that a lot of risk factors associated with Acinetobacter $s p$. infections exist in ICU like opportunity for cross transmission many environment reservoirs, immuno-compromised status of the patients, multiple indwelling devices, etc,.

A study conducted in Karnataka by Aparna Shivaprasad et al., (2014) has documented $100 \%$ strains positive for carbapenemases production by MHT. Similarly, we observed a very high prevalence $(100 \%)$ of enzyme production in our study i.e. the isolates were either carbapenemase or MBL producers. Phenotypically, Modified Hodge Test (MHT) for carbapenemases production confirmed a high prevalence of $86 \%(86 / 100)$ in our isolates, which is quite similar to a study by Duygu Aksoy et al., where 50 strains (96\%) were detected as carbapenemase positive by the modified Hodge test.
In another study by Anil V. Kumar et al., (2011), 71\% of Acinetobacter sps. were found to be carbapenemases producers. This was also in concordance with the results which were obtained by Lee et al., in Korea (2001), where $73 \%$ (59/81) of the isolates were found to be carbapenemase positive by the MHT (Lee et al., 2001). This suggests that carbapenamase producing Acinetobacter $s p$ might be on a rise worldwide which could be due to indiscriminate and overuse of carbapenems in our hospitals. On a contrary although, Noyal et al., in 2009 reported only 1 strain $(14.3 \%)$ positive for carbapenemases production by Modified Hodge test (Noyal et al., 2009). Likewise, another study of same geographical area, by Richa Hans et al., in 2015 documented $26.4 \%$ carbapenemase production and a very low prevalence of $2.96 \%$ was also reported by Patwardhan et al., 2013. A study by Uma et al., (2009) reported $70.9 \%$ MBL production. On a contrary, another study conducted by Noyal et al., in 2008 , only 3 (6.5\%) were MBL producers.

Although there are no established phenotypic methods available for the detection of specific serine carbapenemases. However, for zinc based carbapenemases (MBLs) various methods like EDTA-disc potentiation test, MBL E-test and EDTA based microbiological assay are available (Richa Hans et al, 2015).

All our isolates were thus, further subjected to DPT and EDS simultaneously to detect MBL production. Overall MBL production was found quite significant $88 \%$ (88/100) among our isolates close to a study by Uma et al., (2009) reporting $70.9 \%$ MBL production. Another Indian study by Shanthi et al., (2009) observed $80 \%$ MBL production in nonfermenters. Whereas, on a contrary, in a study conducted in 2007 by Sinha et al, none of the isolates were reported as MBL producers. Dheepa Muthusamy et al., (2012) detected $10 \%$ of the strains to be MBL producers. In a 
similar study conducted by Noyal et al., (2009) in Pondicherry, South India, in the year 2009, 6.5\% MBL producers were identified among Acinetobacter sps.

According to Yong et al., (2002), the imipenem (IMP) $10 \quad \mu \mathrm{g}$-EDTA $750 \quad \mu \mathrm{g}$ combined disc test has $95.7 \%$ sensitivity and $91.0 \%$ specificity for detection of metallo- $\beta$ lactamases in MBL-producing Ps. aeruginosa and Acinetobacter spp. (Yong D et al, 2002).

In our study EDS detected 86\% (86/100) of the strains as MBL producers. This was in concordance to various studies reporting EDS as one of the convenient methods for the detection of Ambler class B MBL production. In 2005, a similar study by Jesudasan et al., reported 72 per cent of the strains to be MBL producers. In 2014, a study by Aparna S et al., documented 67.05 per cent MBL production. By the same method although, in 2011 John S et al., reported only 14.8 per cent MBL production among Acinetobacter sps.

In our study, DPT confirmed only $48 \%$ (48/100) of the isolates as MBL producers. This was contrary to certain studies which revealed $81.18 \%$ and $96.6 \%$ of the strains positive by DPT (Aparna et al., 2014 and Irfan et al, 2008)

Therefore, probably the low potency of commercially available imipenem-EDTA combination discs used in DPT may be a reason of low sensitivity of DPT in the present study. Therefore, according to our study, EDS is found more sensitive $86 \%$ $(86 / 100)$ than DPT $48 \%(48 / 100)$.

\section{Significance of the results}

Modified Hodge Test (MHT) is found to be a reliable phenotypic method for the detection of carbapenemases production. However for the detection of MBL production, EDTA Disc
Synergy (EDS) test is found to be more sensitive and reliable in comparison to Disc Potentiation Test (DPT).

Both ceftazidime and imipenem should be used simultaneously to conduct EDS for avoiding false negative results. Moreover, there is a need of two or more tests to identify all the carbapenemases producing isolates. MBL production is an important mechanism of carbapenem resistance among Acinetobacter sps. in the present study.

Thus, it is recommended to perform these simple phenotypic tests like Modified Hodge Test (MHT) for carbapenemases production and Disc Potentiation test (DPT) and EDTA Disc Synergy (EDS) test for keeping a check on MBL production in small tertiary care centers or in resource limited set-ups to determine resistance mechanisms and prevent the indiscriminate usage of antibiotics. These tests are comparatively easily applicable and economical for screening of enzyme production in Acinetobacter species. and can be incorporated into the routine testing of any busy microbiology laboratory.

Our study concludes that high prevalence of carbapenem resistant Acinetobacter species with MBL production is an alarming situation which needs strict infection control measures.

\section{Abbreviations}

MHT: Modified Hodge Test

EDS: EDTA Disc Synergy (EDS)

DPT: Disc Potentiation Test (DPT)

\section{References}

Aparna Shivaprasad, Beena Antony, Poornima Shenoy. Comparative evaluation of Four Phenotypic Tests for Detection of Metallo- $\beta$-Lactamase and Carbapenemase Production in 
Acinetobacter baumannii; Journal of Clinical and Diagnostic Research. 2014 May, Vol-8(5): DC05-DC08.

Collee JG, Miles RS, Watt B. Identification of bacteria. In: Collee JG, Fraser AG, Marmion BP, Simmons A, editors. Practical Medical Microbiology. 14th edn. Singapore: Churchill Livingstone Publishers, Longman; 2003;131-49.

Giamarellou H, Antoniadou A, Kanellakopoulou K. Acinetobacter baumannii: a universal threat to public health? Int J Antimicrob Agents 2008; 32: 106-19.

Irfan S, Zafar A, Guhar D, Ahsan T, Hasan R. Metallo- $\beta$ - lactamase- producing clinical isolates of Acinetobacter spp and Pseudomonas aeruginosa from intensive care unit patients of a Tertiary care Hospital. Indian J Med Microbiol. 2008; 26(3): 243-5.

Jaggi N. Acinetobacter baumannii isolates J Microbiol Infect Dis. 2012;2(2) June.

Jesudasan MV, Kandathil AJ, Balaji V. Comparison of two methods to detect Carbapenemase and Metallo- $\beta$.lactamase production in clinical isolates. Indian J med Res. 2005; 121:780-3.

John S, Balagurunathan R. Metallo betalactamase producing Pseudomonas aeruginosa and Acinetobacter baumannii. Indian $J$ Med Microbiol. 2011; 29(3):302-4

Krista L, Epp S, Vivika A, Piert M, Lulu K, Karin O, et al., The prevalence and the antibiotic susceptibility of Acinetobacter baumannii, Pseudomonas aeruginosa and Klebsiella pneumonia in Estonian intensive care units in comparison with European data. Scand J Infect Dis 2006;38:1001.

Kumar AV, Pillai VS, Kavitha R, Dinesh, Karim S. The phenotypic detection of carbapenemases in meropenem resistant Acinetobacter calcoaceticusbaumannii complex in a tertiary care hospital in South India. Journal of clinical and diagnostic research. 2011; 5(2): 223-6.

Lee K, Chong Y, Shin HB, Kim YA, Yong D, Yum JH. Modified Hodge and EDTA - disk synergy tests to screen metallo$\beta$-lactamase-producing strains of Pseudomonas and Acinetobacter species. Clin Microbiol Infect 2001; 7: 88-91.

Marchiaro P, Mussi MA, Ballerini V, Pasteran F, Viale AM, Vila AJ, et al., Sensitive EDTA-based micro biological assays for detection of metallo- $\beta$-lactamases in non fermentative gram-negative bacteria. $J$ Clin Microbiol 2005;43:5648-52.

M. Duygu Aksoy, Şaban Çavuşlu, H. Murat Tuğrul. Investigation of Metallo Beta Lactamases and Oxacilinases in Carbapenem Resistant Acinetobacter baumannii Strains Isolated from Inpatients; Balkan Med J 2015;32:7983.

Muneeza Anwar, Hassan Ejaz, Aizza Zafar, and Hamdan Hamid; Phenotypic Detection of Metallo-Beta-Lactamases in Carbapenem Resistant Acinetobacter baumannii Isolated from Pediatric Patients in Pakistan. Journal of Pathogens Volume 2016, Article ID 8603964, 6 pages, 2016.

Muthusamy D, Appalaraju Boppe. Phenotypic methods for the detection of various beta lactamases in carbapenem resistant isolates of Acinetobacter baumanii at a tertiary care hospital in south India. Journal of Clinical and Diagnostic Research [serial online] 2012; 6:970-73.

National Committee for Clinical Laboratory Standards. Performance standards for antimicrobials disc susceptibility tests. Approved Standard, 7th ed. NCCLS 
document M2-A7. Wayne, PA: National Committee for Clinical Laboratory Standards; 2000.

Noyal MJC, Menezes GA, Garish BN, Sujatha S, Parija SC. A simple screening test for the detection of carbapenemases in the clinical isolates of non fermentative, gram negative bacteria. Indian J Med Res 2009;129:707-71.

Patwardhan N Satish, Patwardhan S Shripad \& Deore Santosh; Detection of ESBL, MBL, AmpC and Carbapenemases and their coexistence in clinical isolates of gram negative bacteria; JMRP 2013; 2(7):300.

Payne DJ, Bateson JH, Gasson BC, Proctor D, Khushi T, Farmer TH, et al., Inhibition of metallo- $\beta$-lactamases by a series of mercaptoacetic acid thiol ester derivatives. Antimicrob Agents Chemother 1997;41:135-40.

Richa Hans, Dakshina Bisht, Ritu Agarwal, M. Irfan. Phenotypic detection of mbl, ampc beta-lactamase and carbapenemases in multi drug resistant isolates of Acinetobacter baumannii. Int J Med Res Health Sci. 2015; 4(2): 311-316.

Uma Karthika, R. Srinivasa Rao, Suchismita Sahoo, P. Shashikala, Reba Kanungo, S. Jayachandran and K. Prashanth Phenotypic and genotypic assays for detecting the prevalence of MBL in clinical isolates of Acinetobacter spp. baumannii from a South Indian tertiary care hospital J Medical Microbiology 2009; 58: 430-435.

Shanthi M, Sekar U. Multi-drug resistant Pseudomonas aeruginosa and Acinetobacter baumanii infections among hospitalized patients: Risk factors and outcomes. J Assoc Phys India 2009;57:636-45.

Sinha M, Srinivasa H. Mechanisms of resistance to carbapenems in meropenem resistant Acinetobacter isolates from clinical samples. Indian J Med Microbiol 2007;25:21-5.

Walsh TR, Bolmstrom A, Qwarnstrom A, Gales A. Evaluation of a new Etest for detecting metallo- $\beta$ lactamases in routine clinical testing. $J$ Clin Microbiol 2002; 40: 2755-9.

Walsh TR. The emergence and implications of metallo- $\beta$-lactamases in Gramnegative bacteria. Clin Microbiol Infect 2005; 11(Suppl 6): S2-9.

Yong D, Lee K, Yum JH, Shin HB, Rossolini GM, Chong Y. Imipenem-EDTA disk method for differentiation of metallo$\beta$-lactamase-producing clinical isolates of Pseudomonas spp. and Acinetobacter spp. J Clin Microbiol 2002; 40: 3798-801.

\section{How to cite this article:}

Ankur Goyal, Neha K. Mani, Renu Chahar, Ankita Soni and Sapna Goyal. 2018. Comparison of Various Phenotypic Methods in Detection of Carbapenemases and Metallo-Beta-Lactamases in Carbapenem Resistant Clinical Isolates of Acinetobacter Species at a Tertiary Care Centre in North India. Int.J.Curr.Microbiol.App.Sci. 7(01): 3023-3030.

doi: https://doi.org/10.20546/ijcmas.2018.701.359 